\title{
Gas-induced susceptibility artefacts on diffusion- weighted MRI of the rectum at 1.5 T - Effect of applying a micro-enema to improve image quality
}

Citation for published version (APA):

van Griethuysen, J. J. M., Bus, E. M., Hauptmann, M., Lahaye, M. J., Maas, M., ter Beek, L. C., Beets, G. L., Bakers, F. C. H., Beets-Tan, R. G. H., \& Lambregts, D. M. J. (2018). Gas-induced susceptibility artefacts on diffusion-weighted MRI of the rectum at $1.5 \mathrm{~T}$ - Effect of applying a micro-enema to improve image quality. European Journal of Radiology, 99, 131-137. https://doi.org/10.1016/j.ejrad.2017.12.020

Document status and date:

Published: 01/02/2018

DOI:

10.1016/j.ejrad.2017.12.020

Document Version:

Publisher's PDF, also known as Version of record

Document license:

Taverne

Please check the document version of this publication:

- A submitted manuscript is the version of the article upon submission and before peer-review. There can be important differences between the submitted version and the official published version of record.

People interested in the research are advised to contact the author for the final version of the publication, or visit the DOI to the publisher's website.

- The final author version and the galley proof are versions of the publication after peer review.

- The final published version features the final layout of the paper including the volume, issue and page numbers.

Link to publication

\footnotetext{
General rights rights.

- You may freely distribute the URL identifying the publication in the public portal. please follow below link for the End User Agreement:

www.umlib.nl/taverne-license

Take down policy

If you believe that this document breaches copyright please contact us at:

repository@maastrichtuniversity.nl

providing details and we will investigate your claim.
}

Copyright and moral rights for the publications made accessible in the public portal are retained by the authors and/or other copyright owners and it is a condition of accessing publications that users recognise and abide by the legal requirements associated with these

- Users may download and print one copy of any publication from the public portal for the purpose of private study or research.

- You may not further distribute the material or use it for any profit-making activity or commercial gain

If the publication is distributed under the terms of Article $25 \mathrm{fa}$ of the Dutch Copyright Act, indicated by the "Taverne" license above, 


\title{
Gas-induced susceptibility artefacts on diffusion-weighted MRI of the rectum at $1.5 \mathrm{~T}$ - Effect of applying a micro-enema to improve image quality
}

\author{
Joost J.M. van Griethuysen ${ }^{\mathrm{a}, \mathrm{b}}$, Elyse M. Bus ${ }^{\mathrm{a}}$, Michael Hauptmann ${ }^{\mathrm{c}}$, Max J. Lahaye ${ }^{\mathrm{a}}$, \\ Monique Maas ${ }^{\mathrm{a}}$, Leon C. ter Beek ${ }^{\mathrm{a}}$, Geerard L. Beets ${ }^{\mathrm{b}, \mathrm{d}}$, Frans C.H. Bakers ${ }^{\mathrm{e}}$, \\ Regina G.H. Beets-Tan ${ }^{\mathrm{a}, \mathrm{b}}$, Doenja M.J. Lambregts ${ }^{\mathrm{a}, *}$ \\ a Department of Radiology, The Netherlands Cancer Institute, Amsterdam, The Netherlands \\ ${ }^{\mathrm{b}}$ GROW School of Oncology and Developmental Biology, Maastricht University Medical Center, Maastricht, The Netherlands \\ ${ }^{\mathrm{c}}$ Department of Psychosocial Research and Epidemiology, The Netherlands Cancer Institute, Amsterdam, The Netherlands \\ d Department of Surgery, the Netherlands Cancer Institute, Amsterdam, The Netherlands \\ ${ }^{\mathrm{e}}$ Department of Radiology, Maastricht University Medical Centre, Maastricht, The Netherlands
}

\section{A R T I C L E I N F O}

\section{Keywords:}

Diffusion-weighted imaging

Susceptibility artifacts

Micro-enema

Bowel preparation

Rectal imaging

Rectal cancer

\begin{abstract}
A B S T R A C T
Purpose: Assess whether application of a micro-enema can reduce gas-induced susceptibility artefacts in Singleshot Echo Planar Imaging (EPI) Diffusion-weighted imaging of the rectum at $1.5 \mathrm{~T}$.

Materials and methods: Retrospective analysis of $\mathrm{n}=50$ rectal cancer patients who each underwent multiple DWI-MRIs (1.5 T) from 2012 to 2016 as part of routine follow-up during a watch-and-wait approach after chemoradiotherapy. From March 2014 DWI-MRIs were routinely acquired after application of a preparatory micro-enema (Microlax ; 5 ml; self-administered shortly before acquisition); before March 2014 no bowel preparation was given. In total, 335 scans were scored by an experienced reader for the presence/severity of gasartefacts (on b1000 DWI), ranging from 0 (no artefact) to 5 (severe artefact). A score $\geq 3$ (moderate-severe) was considered a clinically relevant artefact. A random sample of 100 scans was re-assessed by a second independent reader to study inter-observer effects. Scores were compared between the scans performed without and with a preparatory micro-enema using univariable and multivariable logistic regression taking into account potential confounding factors (age/gender, acquisition parameters, MRI-hardware, rectoscopy prior to MRI).

Results: Clinically relevant gas-artefacts were seen in $24.3 \%$ (no micro-enema) vs. $3.7 \%$ (micro-enema), odds ratios were 0.118 in univariable and 0.230 in multivariable regression $(\mathrm{P}=0.0005$ and 0.0291$)$. Mean severity score ( $\pm \mathrm{SD}$ ) was $1.19 \pm 1.71$ (no-enema) vs $0.32 \pm 0.77$ (micro-enema), odds ratios were 0.321 $(\mathrm{P}<0.0001)$ and $0.489(\mathrm{P}=0.0461)$ in uni- and multivariable regression, respectively. Inter-observer agreement was excellent $(\kappa 0.85)$.

Conclusion: Use of a preparatory micro-enema shortly before rectal EPI-DWI examinations performed at $1.5 \mathrm{~T}$ MRI significantly reduces both the incidence and severity of gas-induced artefacts, compared to examinations performed without bowel preparation.
\end{abstract}

\section{Introduction}

Diffusion-weighted imaging (DWI) is nowadays increasingly adopted as an integral part of oncologic imaging protocols. In rectal cancer, DWI has mainly shown its value for response evaluation and follow-up of rectal tumors after chemoradiotherapy, specifically for the discrimination of viable tumor within areas of post-radiation fibrosis [1-5].

The most commonly used sequence for abdominal diffusion imaging is a single-shot Echo Planar Imaging (EPI) sequence. The main benefit from an EPI approach is its short acquisition time, which minimizes the risk of motion artefacts. However, an important drawback is that EPI sequences are prone to susceptibility artefacts, particularly at higher field strengths [6,7]. Susceptibility artefacts are changes or distortions in image signal caused by local magnetic field inhomogeneities, for example due to the presence of metal objects (e.g. hip replacements or surgical clips). In bowel imaging, these artefacts are mainly caused by the presence of gas in the rectal lumen. In a study by Caglic et al. (in prostate MRIs) it was reported that increased rectal gas-distension correlates significantly with reduced DWI image quality and increased

\footnotetext{
* Corresponding author at: Department of Radiology, The Netherlands Cancer Institute, PO Box 90203, 1006 BE Amsterdam, The Netherlands.

E-mail address: d.lambregts@nki.nl (D.M.J. Lambregts).
} 
Table 1

DWI sequences used during the study period.

\begin{tabular}{|c|c|c|c|c|}
\hline Parameter & DWI-1 & DWI-2 & DWI-3 & DWI-4 \\
\hline Repetition Time & $4652-5727$ & $1240-3721$ & $2624-5308$ & $4186-4549$ \\
\hline Echo Time & $70-73$ & $81-104$ & $64-79$ & $69-71$ \\
\hline No. of Slices & 24 & 20 & $20-24$ & 20 \\
\hline FOV $(\mathrm{mm})$ & $320-370$ & $153-180$ & $247-320$ & 320 \\
\hline In-plane Resolution $(\mathrm{mm} \times \mathrm{mm})$ & $1.25 \times 1.25$ & $1.41 \times 1.41$ & $1.25 \times 1.25$ & $1.25 \times 1.25$ \\
\hline Slice Thickness (mm) & 5 & 5 & 5 & 5 \\
\hline Phase encoding direction & $\mathrm{AP}$ & $\mathrm{AP}$ & $\mathrm{AP}$ & AP \\
\hline Parallel imaging (SENSE) Factor & 2 & 2 & 2 & 2 \\
\hline Number of Signals Averaged (NSA) & 5 & 8 & 5 & 5 \\
\hline Flip Angle & 70 & 90 & 70 & 70 \\
\hline Acquisition Matrix & 256 & 128 & 256 & $256-320$ \\
\hline Echo Planar Imaging (EPI) Factor & $55-77$ & $55-99$ & $61-91$ & $61-97$ \\
\hline Fat Saturation Technique & SPIR & SPIR & SPAIR & SPAIR \\
\hline b-values ${ }^{\mathrm{a}}$ & $0,500,1000$ & $0,(500), 1000$ & $0,(25,50,100), 500,1000,(2000)$ & 0,1000 \\
\hline
\end{tabular}

NB. FOV $=$ Field of View, AP = Anterior-Posterior, SPIR $=$ Spectral Presaturation with Inversion Recovery, SPAIR = Spectral Attenuated Inversion Recovery.

${ }^{a}$ Only the b1000 images were used for image evaluation in the current study.

DWI artefacts [8]. Particularly when the bowel itself is the organ under investigation, gas-induced susceptibility artefacts can severely reduce the diagnostic image quality, in some cases even rendering the images non-diagnostic. In published reports on bowel DWI 4-11\% of patients had to be excluded from analyses due to poor DWI scan quality [9-12].

To reduce the influence of these artefacts on image quality, two main strategies can be employed: 1) change the acquisition parameters (i.e. type of DWI sequence) or 2) remove the cause of the artefact.

So far, most published studies have focused on the first approach and tested alternative ways of DWI image acquisition such as parallel imaging [13], smaller Field of View (FOV) [14] or bipolar DWI acquisition [15]. A potential solution to remove the cause of the artefact is to reduce the amount of air in the rectal lumen by rectal filling, where the rectum is filled with a liquid (such as ultrasound gel) prior to image acquisition, replacing the gas. However, a potential downside of this approach is that it causes distension of rectum and compression of the surrounding mesorectal fat $[16,17]$, potentially hampering correct assessment of the relation between the tumor and mesorectal fascia [18]. Use of endorectal filling is therefore not routinely recommended [19].

An alternative potential solution is the application of a preparatory micro-enema shortly prior to image acquisition. A micro-enema can typically be self-administered by the patient to reduce the amount of gas (and stool) in the rectum.

The aim of this study was to test this hypothesis and investigate to what extend the use of a micro-enema can reduce the amount of gasinduced susceptibility artefacts on EPI-DWI of the rectum.

\section{Materials and methods}

The study was approved by the local institutional review board. Due to the retrospective nature of the study, informed consent was not required.

\subsection{Patients}

We retrospectively selected 50 consecutive rectal cancer patients (66\% male, mean age 63) who each underwent serial MR imaging including an EPI-DWI sequence of the rectum as part of their routine follow-up during a 'watch-and-wait' policy between January 2012 and February 2016 at Maastricht Univeristy Medical Center (MUMC). All patients had previously been treated with long-course chemoradiotherapy and were non-operatively managed due to strong clinical evidence of a clinical complete response. The follow-up protocol included regular MRI performed 3 monthly in the first year and 6 monthly in the second to fifth year of follow-up. In March 2014, use of a rectal micro-enema (Microlax', McNeil Healthcare, Ireland) was introduced into the routine protocol. Inclusion criteria consisted of: 1) availability of at least 2 consecutive follow-up MRIs including a EPIDWI sequence, with at least 1 MRI without bowel preparation and 1 MRI after application of a micro-enema, 2) no treatment (radiation or surgery) performed between the various sequential scans, 3) no history of hip replacement surgery (as hip prostheses will results in artefacts on DWI, as a result of which the presence of air artefacts cannot be sufficiently studied).

\subsection{Image acquisition and patient preparation}

All MR images were acquired on a 1.5T MR system (Intera (Achieva) or Ingenia MR system; Philips Healthcare, Best, The Netherlands) using a phased-array body coil. The routine protocol included T2-weighted turbo spin echo sequences in 3 planes (sagittal, axial and coronal) and an axial EPI-DWI sequence with $b=1000$ being the highest $b$-factor. The transverse T2-weighted and DW-sequences were angled perpendicular to the former tumor axis (i.e. the fibrotic remnant) as visualized on the sagittal planning scan. Image angulation was consistent over time for the various follow-up scans. Detailed sequence parameters of the DWI-sequences used during the study period are provided in Table 1 . For the scans performed with a preparatory micro-enema (from March 2014), the micro-enema consisted of a $5 \mathrm{ml}$ solution, that was self-administered by the patients \pm 15 min prior to acquisition. Apart from the micro-enema no bowel preparation or spasmolytic agents were applied.

\subsection{Image assessment}

In total 335 scans (in 50 patients) were analyzed by an experienced reader (D.L.) who scored the presence and severity of gas-related susceptibility artefacts using a 6-point score $(0=$ no artefact, $1=$ mild artefact, $2=$ mild - moderate artefact, $3=$ moderate artefact, $4=$ moderate - severe artefact, $5=$ severe artefact). The scoring system with representative imaging examples is illustrated in Fig. 1. A second experienced reader (J.G.) independently analyzed a random sample of $n=100$ scans (50 scans without preparation, 50 scans after application of a micro-enema) using the same scoring system to study inter-observer effects. Both readers were blinded to clinical patient data, whether or not the patient had undergone a preparatory microenema and to each other's results.

\subsection{Statistical analysis}

The study outline is graphically illustrated in Fig. 2: to assess the effect of the introduction of the preparatory rectal micro-enema on DW 
A. no artefact
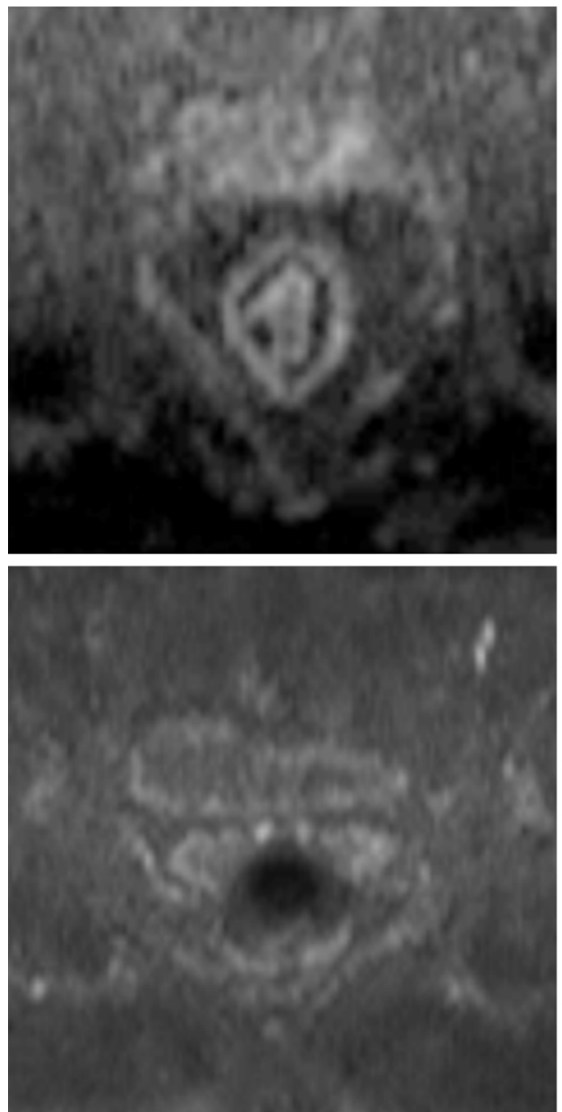

D. moderate
B. mild
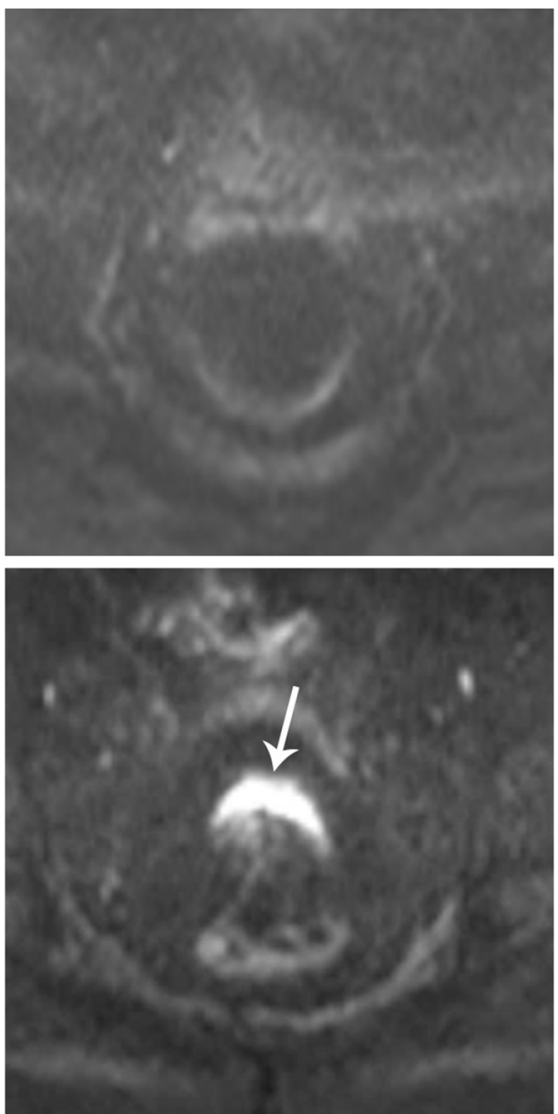

E. moderate-severe
C. mild-moderate
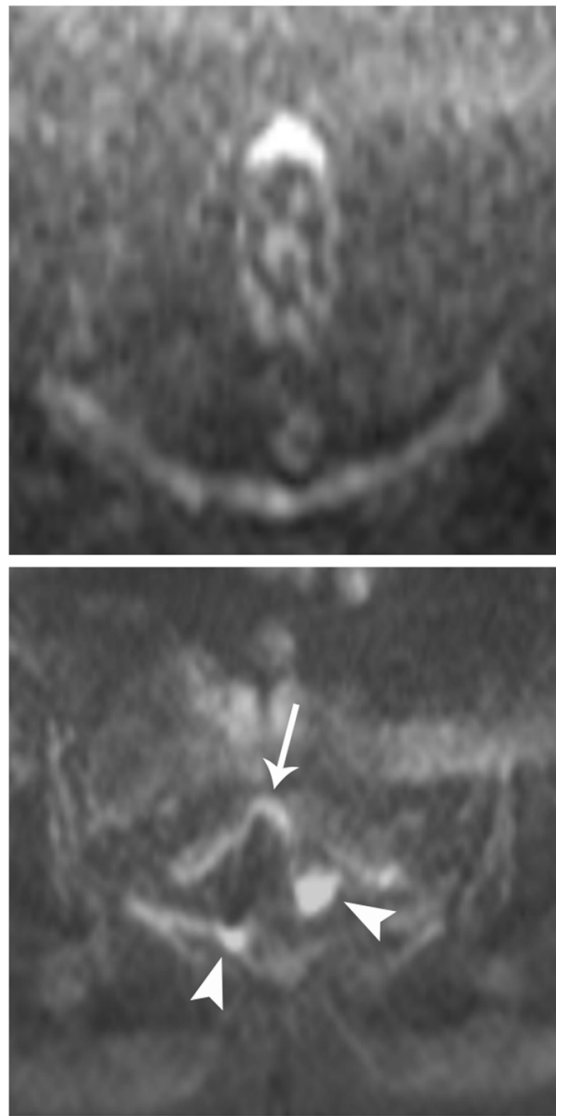

F. severe

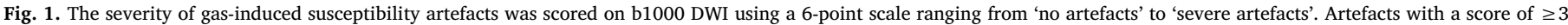

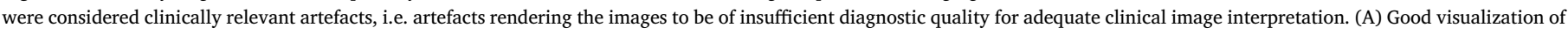

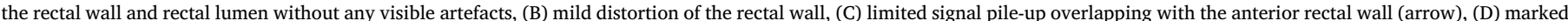

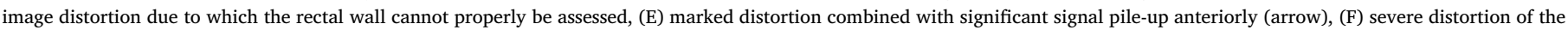
rectal wall (arrow) with severe signal pile-up (arrowheads).

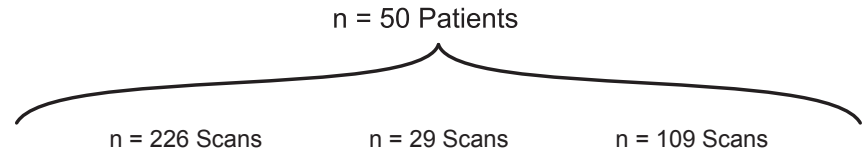

\begin{tabular}{|l|l|l|}
\hline No preparation & $\begin{array}{c}\text { Transition } \\
\text { period }\end{array}$ & $\begin{array}{c}\text { Preparation with } \\
\text { mirco-enema }\end{array}$ \\
\hline & &
\end{tabular}

introduction of micro-enema

February 2016

Fig. 2. Study outline. Fifty patients were included. In these patients, 226 scans were acquired from January 2012 to March 2014 without any bowel preparation (the no micro-enema group). From July 2014 to February 2016, 109 scans were acquired with a preparatory micro-enema (the micro-enema group). Scans made in the transition period after the introduction of the micro-enema (March 2014-July 2014; $n=29$ ) were excluded from the analysis.

image quality, the presence and severity of gas-related artefacts were compared between the scans acquired between January 2012 and March 2014 (='without micro-enema') and scans acquired between July 2014 and February 2016 (='with micro-enema'). Scans acquired between March 2014 and July 2014 (the transit period) were excluded from the analyses as in this period some patients may not yet have received the micro-enema routinely. Primary outcome was the proportion of 'clinically relevant' artefacts in the no micro-enema versus micro-enema scans. Artefacts were considered to be clinically

significant if they would considerably hamper clinical DW image interpretation and were defined for the purpose of this study as artefacts with a severity score of $\geq 3$ (moderate, moderate-severe and severe). The artefact severity score itself was assessed as a secondary outcome. The influence of potential confounding factors was assessed using univariable and multivariable logistic regression with a binary outcome for the primary outcome (clinically relevant artefact yes/no) and ordinal logistic regression for the secondary outcome (6-point severity score). Generalized linear models with generalized estimating equations for clustered data were applied, with scans clustered by patient. The working correlation was exchangeable for the binary logistic regression [20] and independent for the ordinal logistic regression. A $P$ value of $<0.05$ was considered significant. Results for the binary logistic regression differed very little when an independent working correlation was used. Inter-observer agreement was assessed using quadratic weighted Cohen's Kappa $(0-0.20=$ poor, $0.21-0.40=$ fair, $0.41-0.60=$ moderate, $0.61-0.80=$ good and $0.81-1.00=$ excellent agreement). Statistical analysis was performed using the Statistical Package for the Social Sciences (SPSS version 23.0, IBM ${ }^{\circ}$ SPSS $^{\circ}$ Inc. Chicago, IL) and Statistics Analysis Software (SAS version 9.4, SAS ${ }^{\circ}$ Institute Inc., Cary, NC). 
Table 2

Baseline characteristics and results for the scans performed without and with a preparatory micro-enema.

\begin{tabular}{|c|c|c|c|c|c|c|}
\hline $\begin{array}{l}\text { Baseline characteristics (at the time of image } \\
\text { acquisition) }\end{array}$ & No Enema $\mathrm{N}=226$ scans & Enema $\mathrm{N}=109$ scans & Results & & $\begin{array}{l}\text { No Enema } \mathrm{N}=226 \\
\text { scans }\end{array}$ & $\begin{array}{l}\text { Enema } N=109 \\
\text { scans }\end{array}$ \\
\hline \multirow[t]{2}{*}{ Mean age $( \pm S D)$} & $63.4( \pm 11.9)$ & $65.5( \pm 12.2)$ & Clinically & elevant artefacts & & \\
\hline & & & & Yes & $55(24.3 \%)$ & $4(3.7 \%)$ \\
\hline \multicolumn{3}{|l|}{ Gender } & & No & $171(75.7 \%)$ & $105(96.3 \%)$ \\
\hline Male & $143(63.3 \%)$ & $70(64.2 \%)$ & & & & \\
\hline \multirow[t]{2}{*}{ Female } & $83(36.7 \%)$ & $39(35.8 \%)$ & Severity & & & \\
\hline & & & & None & $131(58.0 \%)$ & $86(78.9 \%)$ \\
\hline \multicolumn{3}{|l|}{ MRI-Hardware } & & Mild & $29(12.8 \%)$ & $17(15.6 \%)$ \\
\hline Philips Intera (Achieva) & $113(50 \%)$ & $28(25.7 \%)$ & & Mild-Moderate & $11(4.9 \%)$ & $2(1.8 \%)$ \\
\hline \multirow[t]{2}{*}{ Philips Ingenia } & $113(50 \%)$ & $81(74.3 \%)$ & & Moderate & $19(8.4 \%)$ & $2(1.8 \%)$ \\
\hline & & & & Moderate-Severe & $18(8.0 \%)$ & $2(1.8 \%)$ \\
\hline \multicolumn{2}{|l|}{ DWI sequence (see Table 1) } & & & Severe & $18(8.0 \%)$ & $0(0.0 \%)$ \\
\hline DWI-1 & $51(22.6 \%)$ & $0(0.0 \%)$ & & & & \\
\hline DWI-2 & $60(26.6 \%)$ & $77(70.6 \%)$ & & & & \\
\hline DWI-3 & $115(50.9 \%)$ & $6(5.5 \%)$ & & & & \\
\hline DWI-4 & $0(0.0 \%)$ & $26(23.9 \%)$ & & & & \\
\hline \multicolumn{7}{|l|}{ Rectoscopy prior to MRI } \\
\hline Yes & $137(60.6 \%)$ & $59(54.1 \%)$ & & & & \\
\hline No & $89(39.4 \%)$ & $50(45.9 \%)$ & & & & \\
\hline \multicolumn{7}{|l|}{ Primary tumor height } \\
\hline Low $(<5 \mathrm{~cm})$ & $148(65.5 \%)$ & $71(65.1 \%)$ & & & & \\
\hline Mid-High $(\geq 5 \mathrm{~cm})$ & $78(34.5 \%)$ & $38(34.9 \%)$ & & & & \\
\hline
\end{tabular}

NB. Numbers are absolute numbers, unless otherwise indicated percentages are given in parentheses. SD = Standard deviation

\section{Results}

\subsection{Baseline characteristics}

Table 2 shows the baseline study characteristics. The 50 study patients together underwent a total of 364 DWI-MRI examinations. Twenty-nine scans from the transit period after the introduction of the micro-enema (March 2014-June 2014) were excluded. This left a total of 335 scans for analysis (mean 6.7 examinations per patient, range $2-10)$, of which $226(67.5 \%)$ were acquired without a micro-enema (before March 2014) and 109 (32.5\%) were acquired with a microenema (after June 2014).

\subsection{Intersobserver agreement}

Agreement between the two readers for the use of the 6-point artefact severity score (illustrated in Fig. 1) was excellent with a weighted Kappa of 0.85 (95\% confidence interval 0.77-0.94).

\subsection{Effect of micro-enema on DW image quality}

Fig. 3 compares the number of clinically relevant artefacts and the severity of artefacts between the group of scans without and with a micro-enema. In the group without a micro-enema, clinically relevant artefacts (severity score $\geq 3$ ) occurred in 55/226 (24.3\%) scans versus $4 / 109$ scans $(3.7 \%)$ in the group with a micro-enema (Fig. 3a). The reduction in the number of clinically relevant artefacts was significant with an odds ratio $(\mathrm{OR})$ of $0.118(\mathrm{P}=0.0005)$ in univariable binary logistic regression analysis and an OR of $0.230(\mathrm{P}=0.029)$ in multivariable analysis. The overall artefact severity score was also higher in the group without a micro-enema (Fig. 3b), with a mean severity score of $1.19( \pm 1.71)$, compared to $0.32( \pm 0.77)$ in the scans acquired with a micro-enema. The reduction in the severity of artefacts was significant with an OR of $0.321(P<0.0001)$ in univariable ordinal logistic regression analysis and an OR of $0.489(P=0.046)$ in multivariable analysis.

Of all available variables to adjust for confounding, b-values, acquisition matrix size, flip angle, Number of Signals Averaged, Field of View (FOV) right-left versus anterior-posterior and water-fat shift were excluded because they were either collinear or highly correlated with another variable (correlation coefficient $>0.8$ ). The remaining variables (gender, age at scan time, repetition time, echo time, FOV cranialcaudal, EPI factor, fat saturation technique, MRI-hardware, and flexible rectoscopy performed $<12 \mathrm{~h}$ prior to MRI) were included in all multivariable models. When these variables, including those excluded due to high correlation, were added to a model with the micro-enema effect one by one, no substantial confounding was observed (Table 3). In addition, a sub-analysis was performed to compare the effects of the
A

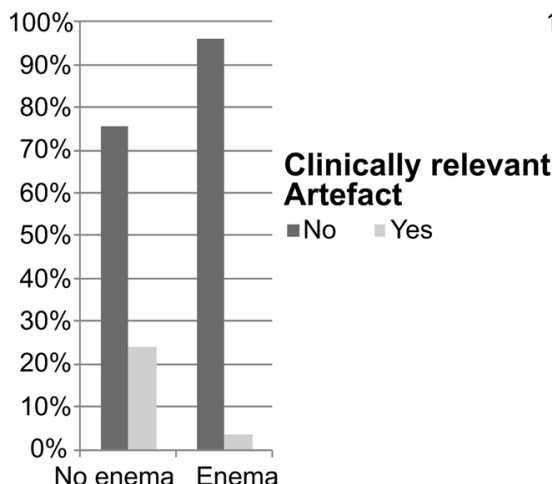

B

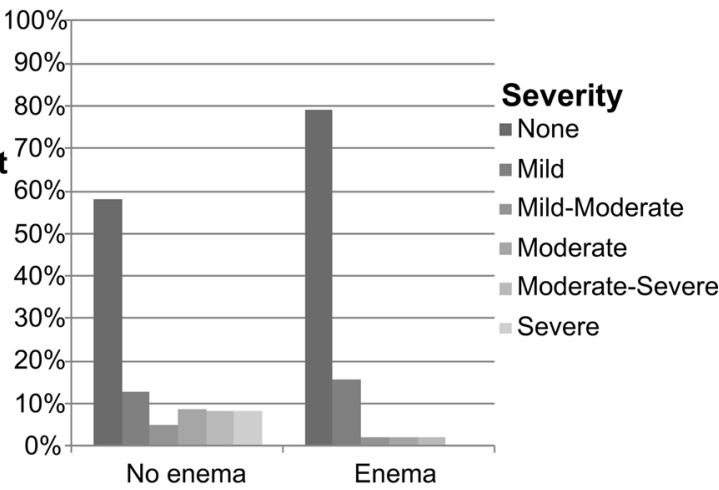

Fig. 3. Boxplots comparing the presence of clinically relevant artefacts (A) and the artefact severity score (B) between the scans acquired without bowel preparation and after application of a preparatory micro-enema. 
Table 3

Results from uni- and multivariable logistic regression.

\begin{tabular}{|c|c|c|c|c|}
\hline & \multicolumn{2}{|c|}{ Presence of clinically relevant artefacts ${ }^{\mathrm{a}}$} & \multicolumn{2}{|l|}{ Severity of artefacts ${ }^{b}$} \\
\hline & Odds ratio $(95 \% \mathrm{CI})$ & P-value & Odds ratio $(95 \% \mathrm{CI})$ & P-value \\
\hline \multicolumn{5}{|l|}{ Effect of preparatory micro-enema } \\
\hline Univariable & $0.118(0.035-0.396)$ & 0.0005 & $0.321(0.193-0.533)$ & $<0.0001$ \\
\hline Multivariable & $0.230(0.061-0.861)$ & 0.0291 & $0.489(0.242-0.988)$ & 0.0461 \\
\hline \multicolumn{5}{|l|}{ Effect of potential confounders } \\
\hline Age $^{*}$ & $0.982(0.950-1.016)$ & 0.3069 & $0.995(0.971-1.020)$ & 0.7110 \\
\hline Gender" & $0.687(0.317-1.489)$ & 0.3413 & $0.591(0.349-1.003)$ & 0.0511 \\
\hline Rectoscopy $^{*}$ & $1.643(0.913-2.958)$ & 0.0978 & $1.478(0.890-2.454)$ & 0.1309 \\
\hline MRI hardware system ${ }^{* c}$ & $0.971(0.567-1.661)$ & 0.9143 & $0.996(0.647-1.535)$ & 0.9864 \\
\hline Repetition Time ${ }^{*}$ & $1.000(1.000-1.001)$ & 0.1468 & $1.000(1.000-1.000)$ & 0.1951 \\
\hline Echo Time ${ }^{*}$ & $0.973(0.941-1.006)$ & 0.1049 & $0.986(0.958-1.015)$ & 0.3261 \\
\hline Flip Angle & $0.610(0.334-1.112)$ & 0.1064 & $0.726(0.449-1.173)$ & 0.1912 \\
\hline Number of Signals Averaged & $0.610(0.334-1.112)$ & 0.1064 & $0.726(0.449-1.173)$ & 0.1912 \\
\hline Water-Fat Shift & $1.085(0.981-1.199)$ & 0.1134 & $1.075(0.978-1.183)$ & 0.1347 \\
\hline Echo Planar Imaging (EPI) Factor" & $1.007(0.966-1.050)$ & 0.7272 & $1.016(0.982-1.050)$ & 0.3660 \\
\hline Fat Saturation Technique ${ }^{* d}$ & $0.861(0.508-1.462)$ & 0.5801 & $0.880(0.542-1.429)$ & 0.6051 \\
\hline Field of View size (mm), anterior-posterior & $1.005(1.000-1.010)$ & 0.0562 & $1.003(0.998-1.007)$ & 0.2204 \\
\hline Field of View size $(\mathrm{mm})$, cranial-caudal ${ }^{*}$ & $0.994(0.985-1.003)$ & 0.1605 & $1.000(0.994-1.005)$ & 0.8626 \\
\hline Field of View size (mm), right-left & $1.004(0.999-1.008)$ & 0.0908 & $1.002(0.999-1.006)$ & 0.2239 \\
\hline Number of b values acquired ${ }^{e}$ & $1.057(0.871-1.284)$ & 0.5715 & $1.028(0.858-1.232)$ & 0.7647 \\
\hline
\end{tabular}

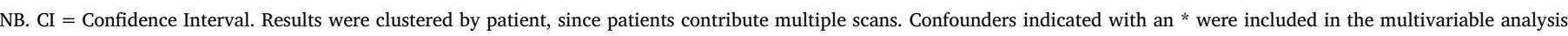

${ }^{a}$ Binary logistic regression.

b Ordinal logistic regression.

${ }^{c}$ All scans were performed on 1.5T using either a Intera (Achieva) or Ingenia MRI system from Philips.

${ }^{\mathrm{d}}$ All scans were performed using either Spectral Presaturation with Inversion Recovery (SPIR) or Spectral Attenuated Inversion Recovery (SPAIR).

e Scans were performed with 2, 3, 6 or 7 b-values, the highest b-value (used for evaluation) always being b1000, see Table 1 .

micro-enema between the scans from patients who originally had a tumor in the distal rectum (i.e. $<5 \mathrm{~cm}$ from the anorectal junction) versus the scans from patients who originally had a tumor in the midhigh rectum ( $\geq 5 \mathrm{~cm}$ from the anorectal junction). This revealed no significant differences between the two groups with respect to the effect of the micro-enema on the proportion of 'clinically relevant' (severity score $\geq 3$ ) artefacts, $P=0.948$. Furthermore, overall artefact severity scores were comparable between the two groups $(1.17$ ( \pm 1.73$)$ in the distal versus $1.24( \pm 1.71)$ in the mid-high rectum group for the scans performed without a micro-enema, and $0.35( \pm 0.88$ ) versus 0.26 ( \pm 0.5 ) for the scans performed with a micro-enema, respectively).

Detailed results of the uni- and multivariable analyses are provided in Table 3. A representative example of how gas-related artefacts reduced over time after the introduction of the routine use of a microenema is provided in Fig. 4 for a patient scanned both without preparation and after application of a micro-enema.

\section{Discussion}

The results of our study show that the application of a preparatory micro-enema shortly before image acquisition significantly reduces both the incidence as well as the severity of gas-induced susceptibility on rectal DWI performed at $1.5 \mathrm{~T}$. The use of a micro-enema reduces the proportion of rectal DWI scans suffering from clinically relevant artefacts - i.e. artefacts that hamper clinical image interpretation - from 1 in every 4 scans to 1 in every 20 scans, thereby offering a substantial potential clinical benefit in terms of improved diagnostic image quality.

To our knowledge only one previous study specifically reported on the use of an enema for the reduction of susceptibility artefacts on DWI. In this study by Lim et al. [21], diagnostic image quality of multiparametric MRI of the prostate was compared at $3.0 \mathrm{~T}$ in patients without bowel preparation versus patients who were instructed to selfadminister an enema on the morning of the day they received their MRI examination. Interestingly, while application of an enema resulted in significantly less stool and gas in the rectum, no significant difference was found in the diagnostic quality of the enema group compared to the no-preparation group. These seemingly conflicting results may be in part caused by the small number of scans assessed ( $n=60$, compared to over 300 scans in the current report), with a relatively low number of events (severe artefacts) ranging from 1 to 7 per group. Another possible explanation is the timing of the enema, which was self-administered in the morning of the day of the examination in the study by Lim et al., compared to 15-30 min prior to examination in our study. Finally the target organ under investigation in the study of Lim was the prostate and not the rectum itself. Various alternative methods to reduce artefacts have been reported, most of which focus on making the EPI sequences more robust. An example is the use of parallel imaging techniques. Parallel imaging allows for less phase-encoding steps, thereby reducing both sensitivity to artefacts and acquisition time [13]. In our study parallel imaging was also employed (factor 2.0). An alternative option is reducing the FOV, allowing for a higher spatial resolution in the phase-encoding direction, where the EPI sequence is most influenced by the susceptibility artefacts. Korn et al. [14] assessed the use of reduced FOV-excitation in DWI for prostate cancer detection using a 5 point scale for image distortion and showed that the reduced FOV significantly reduced image distortion scores by $0.48-0.56$ points. Thian et al. [22] explored the use of a read-out segmented (rs) EPI Sequence, in which the k-space is filled in several segments (as opposed to a standard single shot technique). In $\mathrm{n}=30$ pelvic DWI examinations, lesion conspicuity was significantly better and geometric distortions significantly less on the rs-EPI sequence. The reported magnitude of effect of these technical alterations was less that of the use of a preparatory micro-enema (combined with parallel imaging) in our study. Although with this approach we could already reduce the number of clinically relevant artefacts to less than $5 \%$, future research should focus on further optimizing scan quality by combining patient preparation with artefact reduction acquisition techniques such as the examples described above to ultimately offer the best possible DWI protocols with stable and robust diagnostic image quality, on which radiologists may truly rely on for diagnostic decision making. Although we did not perform formal questionnaires to objectively quantify the degree of patient discomfort for the self-administration of the microenema, in our experience patients tolerated it very well and considered it a minimal extra burden. 
A
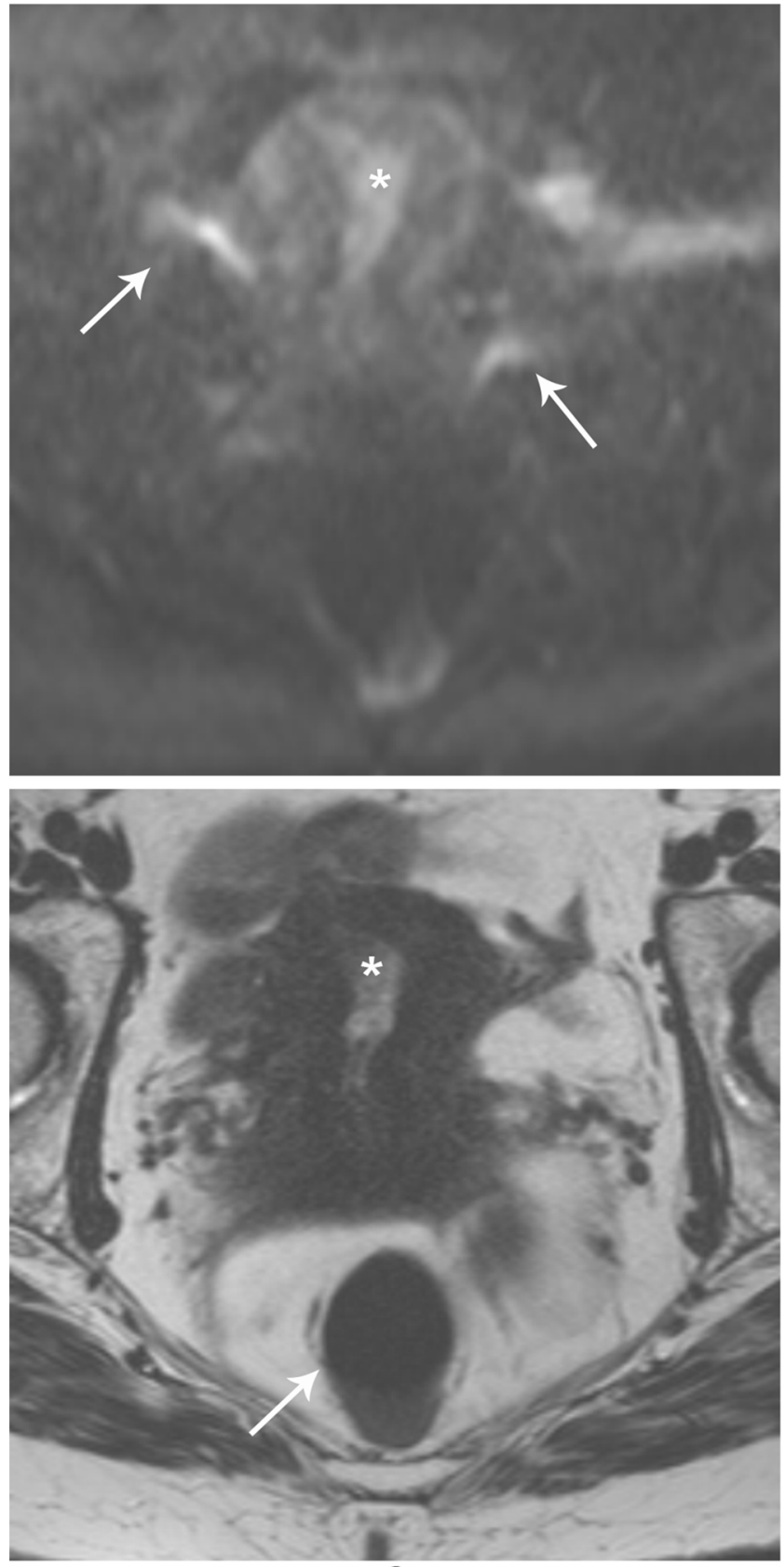

C
B
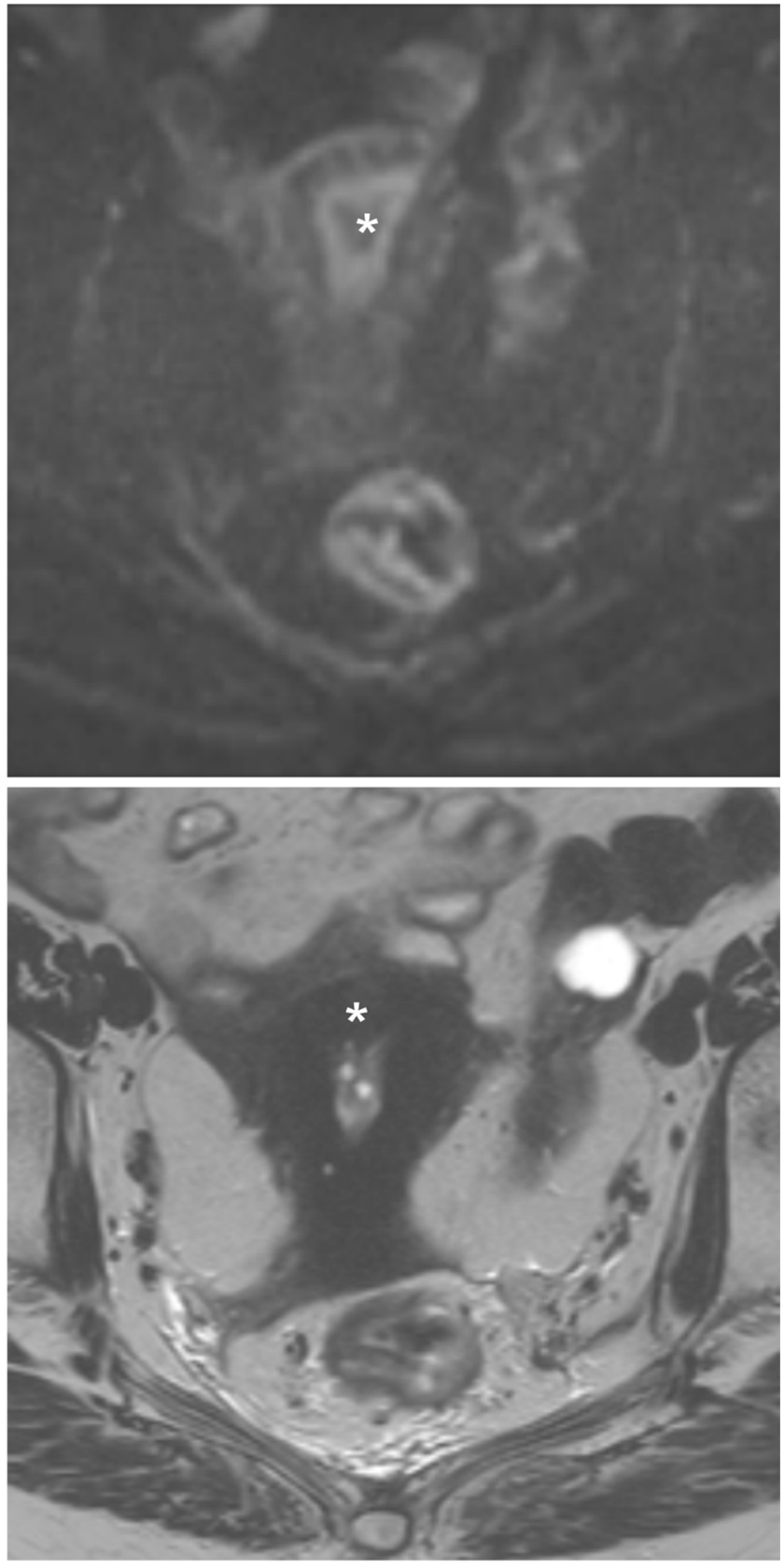

D

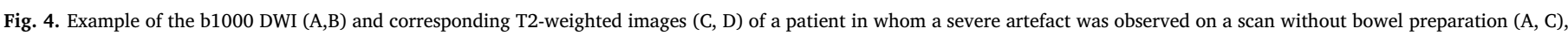

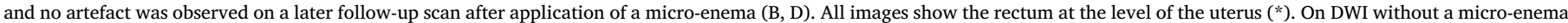

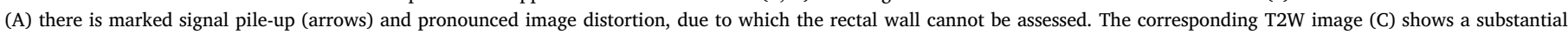

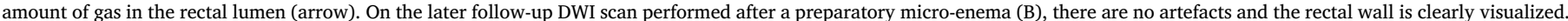
On the corresponding T2W image (D) there is much less gas present within the rectal lumen following the micro-enema.

There are some limitations to our study design. First, we fully acknowledge that an important drawback of our study design is that none of the study patients had rectal tumors in situ at the time of image acquisition, making it impossible to study effects of artefact reduction on lesion conspicuity. All MRIs were performed in patients with a clinical complete response who underwent multiple follow-up MRIs (3-6-monthly) for a longer time period. We chose this approach as a first exploratory step as it offered the benefit of a consistent clinical setting (in which patients underwent no therapeutic procedures in between scans that may influence image quality) which creates the unique opportunity to perform within patient comparisons, thereby reducing effects of interpatient variability. The obvious next step will be to study the clinical benefit of our approach in the staging and restaging setting to determine effects on lesion conspicuity and staging outcomes. Second, all MRIs were performed at $1.5 \mathrm{~T}$. It would be interesting to see how the results of our study translate to $3.0 \mathrm{~T}$ where susceptibility effects will typically be more severe [23] and the gain of applying a micro-enema may thus be more profound. Third, a substantial number of patients underwent flexible rectoscopy just prior to the MRI, because they were in a follow-up protocol that included MRI and rectoscopy on the same day. During this procedure gas is introduced into the rectum, which may have negative effects on MR image quality when performed 
shortly afterwards. On the other hand, the endoscopists in our center typically perform a de-sufflation of the rectum, whilst removing the endoscope which will reduce the amount of gas in the lumen. Moreover, the fact that patients underwent rectoscopy just before their MRI did not have a significant confounding effect in our statistical analyses. Finally, the artefacts in this study were assessed using a more or less subjective scoring system focusing on overall diagnostic image quality. In order to reduce subjectivity we however used a standardized 6-point scoring system which led to excellent interobserver agreement between two independent readers ( $\kappa 0.85$ ).

In conclusion, the use of a preparatory micro-enema shortly prior to image acquisition significantly reduces both the incidence and severity of gas-related susceptibility artefacts in DWI of the rectum performed at 1.5 T. A preparatory micro-enema can easily be self-administered just minutes before the MR examination. As such we believe that - when DWI forms an integral part of the imaging assessment of the rectum - a micro-enema should be considered, as it provides a significant benefit to the image quality at a relatively small cost in terms of preparation time and patient discomfort.

\section{Funding}

This research did not receive any specific grant from funding agencies in the public, commercial, or not-for-profit sectors. No funding was received for this study.

\section{References}

[1] S.X. Rao, M.S. Zeng, C.Z. Chen, et al., The value of diffusion-weighted imaging in combination with T2-weighted imaging for rectal cancer detection, Eur. J. Radiol. 65 (2008) 299-303.

[2] S.H. Kim, J.M. Lee, S.H. Hong, et al., Locally advanced rectal cancer: added value of diffusion-weighted MR imaging in the evaluation of tumor response to neoadjuvant chemo- and radiation therapy, Radiology 253 (2009) 116-125.

[3] D.M.J. Lambregts, V. Vandecaveye, B. Barbaro, et al., Diffusion-weighted MRI for selection of complete responders after chemoradiation for locally advanced rectal cancer: a multicenter study, Ann. Surg. Oncol. 18 (2011) 2224-2231.

[4] S.H. Kim, J.M. Lee, S.H. Hong, et al, Locally advanced rectal cancer: added value of diffusion-weighted MR imaging in the evaluation of tumor response to neoadjuvant chemo- and radiation therapy, Radiology 253 (2009) 116-125.

[5] D.M. Lambregts, M.J. Lahaye, L.A. Heijnen, et al., MRI and diffusion-weighted MRI to diagnose a local tumour regrowth during long-term follow-up of rectal cancer patients treated with organ preservation after chemoradiotherapy, Eur. Radiol. 26 (July (7)) (2016) 2118-2125.

[6] D. Le Bihan, C. Poupon, A. Amadon, F. Lethimonnier, Artifacts and pitfalls in diffusion MRI, J. Magn. Reson. Imaging 24 (2006) 478-488.

[7] D.-M. Koh, T. Takahara, Y. Imai, D.J. Collins, Practical aspects of assessing tumors using clinical diffusion-weighted imaging in the body, Magn. Reson. Med. Sci. 6 (2007) 211-224.

[8] I. Caglic, N.L. Hansen, R.A. Slough, A.J. Patterson, T. Barrett, Evaluating the effect of rectal distension on prostate multiparametric MRI image quality, Eur. J. Radiol. 90 (2017) 174-180.

[9] F. Regini, S. Gourtsoyianni, R. Cardoso De Melo, et al., Rectal tumour volume (GTV) delineation using T2-weighted and diffusion-weighted MRI: Implications for radiotherapy planning, Eur. J. Radiol. 83 (2014) 768-772.

[10] I.M. Blazic, G.B. Lilic, M.M. Gajic, Quantitative assessment of rectal cancer response to neoadjuvant combined chemotherapy and radiation therapy: comparison of three methods of positioning region of interest for ADC measurements at diffusionweighted MR imaging, Radiology (2016) 151908.

[11] M.H. Choi, S.N. Oh, S.E. Rha, et al., Diffusion-weighted imaging: apparent diffusion coefficient histogram analysis for detecting pathologic complete response to chemoradiotherapy in locally advanced rectal cancer, J. Magn. Reson. Imaging 44 (2016) 212-220.

[12] P.V. Foti, G. Privitera, S. Piana, et al., Locally advanced rectal cancer: qualitative and quantitative evaluation of diffusion-weighted MR imaging in the response assessment after neoadjuvant chemo-radiotherapy, Eur. J. Radiol. Open 3 (2016) $145-152$.

[13] K. Nasu, Y. Kuroki, S. Kuroki, K. Murakami, S. Nawano, N. Moriyama, Diffusionweighted single shot echo planar imaging of colorectal cancer using a sensitivityencoding technique, Jpn. J. Clin. Oncol. 34 (2004) 620-626.

[14] N. Korn, J. Kurhanewicz, S. Banerjee, O. Starobinets, E. Saritas, S. Noworolski, Reduced-FOV excitation decreases susceptibility artifact in diffusion-weighted MRI with endorectal coil for prostate cancer detection, Magn. Reson. Imaging 33 (2015) $56-62$.

[15] S. Kyriazi, M. Blackledge, D.J. Collins, N.M. Desouza, Optimising diffusionweighted imaging in the abdomen and pelvis: comparison of image quality between monopolar and bipolar single-shot spin-echo echo-planar sequences, Eur. Radiol. 20 (2010) 2422-2431.

[16] H. Kaur, H. Choi, Y.N. You, et al., MR imaging for preoperative evaluation of primary rectal cancer: practical considerations, Radiographics 32 (2012) 389-409.

[17] A. Dal Lago, A.E. Minetti, P. Biondetti, M. Corsetti, G. Basilisco, Magnetic resonance imaging of the rectum during distension, Dis. Colon Rectum 48 (2005) 1220-1227.

[18] A. Slater, S. Halligan, S.A. Taylor, Marshall M: Distance between the rectal wall and mesorectal fascia measured by MRI: Effect of rectal distension and implications for preoperative prediction of a tumour-free circumferential resection margin, Clin. Radiol. 61 (2006) 65-70.

[19] R.G. Beets-Tan, D.M. Lambregts, M. Maas, et al., Magnetic resonance imaging for the clinical management of rectal cancer patients: recommendations from the 2012 European Society of Gastrointestinal and Abdominal Radiology (ESGAR) consensus meeting, Eur. Radiol. 23 (2013) 2522-2531.

[20] K.-Y. Liang, S.L. Zeger, Longitudinal data analysis using generalized linear models, Biometrika 73 (1986) 13-22.

[21] C. Lim, J. Quon, M. McInnes, W.M. Shabana, M. El-Khodary, N. Schieda, Does a cleansing enema improve image quality of 3T surface coil multiparametric prostate MRI? J. Magn. Reson. Imaging 42 (2015) 689-697.

[22] Y.L. Thian, W. Xie, D.A. Porter, B. Weileng Ang, Readout-segmented echo-planar imaging for diffusion-weighted imaging in the pelvis at 3T-A feasibility study, Acad. Radiol. 21 (2014) 531-537.

[23] P. Riffel, R.K. Rao, S. Haneder, M. Meyer, S.O. Schoenberg, H.J. Michaely, Impact of field strength and RF excitation on abdominal diffusion-weighted magnetic resonance imaging, World J. Radiol. 5 (2013) 334-344. 